\title{
Darstellung, ${ }^{11} \mathrm{~B}$-NMR- und Schwingungsspektren der oktaedrischen closo-Boratanionen $\mathrm{B}_{6} \mathrm{X}_{6}{ }^{2-} ; \mathrm{X}=\mathrm{H}, \mathrm{Cl}, \mathrm{Br}, \mathrm{I}$
}

\author{
Preparation, ${ }^{11} \mathrm{~B}$ NMR and Vibrational Spectra \\ of the Octahedral closo-Borate Anions $\mathrm{B}_{6} \mathrm{X}_{6}{ }^{2-} ; \mathrm{X}=\mathrm{H}, \mathrm{Cl}, \mathrm{Br}, \mathrm{I}$ \\ W. Preetz* und J. Fritze \\ Institut für anorganische Chemie der Christian-Albrechts-Universität, \\ Olshausenstraße 40, D-2300 Kiel
}

Z. Naturforsch. 39b, 1472-1477 (1984); eingegangen am 14. Juni 1984

Hexahalogenohexaborates, ${ }^{11}$ B NMR Spectra, IR Spectra, Raman Spectra

On treatment of $\mathrm{Na}_{2} \mathrm{~B}_{6} \mathrm{H}_{6}$ with halogens in aqueous alkaline solution, the hexahalogenoborates $\mathrm{B}_{6} \mathrm{X}_{6}{ }^{2-}, \mathrm{X}=\mathrm{Cl}, \mathrm{Br}, \mathrm{I}$, are formed. Their octahedral structure is proved by ${ }^{11} \mathrm{~B} \mathrm{NMR}$ spectroscopy. The chemical shifts in relation to $\mathrm{BF}_{3} \cdot \mathrm{OEt}_{2}$ increase within the series $\mathrm{H}(-13.5)<\mathrm{Cl}$ $(-17.4)<\mathrm{Br}(-18.5)<\mathrm{I}(-27.5 \mathrm{ppm})$. The IR and Raman spectra are assigned according to point group $\mathrm{O}_{\mathrm{h}}$. The internal vibrations of the $\mathrm{B}_{6}$-cage, observed in the range $750-1250 \mathrm{~cm}^{-1}$ at nearly unchanged positions, are distinguishable from vibrations mainly originating from motions of the $\mathrm{X}_{6}$-shell against the rigid $\mathrm{B}_{6}$-cluster.

\section{Einleitung}

Das kleinste bisher bekannte closo-Hydroboratanion $\mathrm{B}_{6} \mathrm{H}_{6}{ }^{2-}$ ist 1964 erstmals dargestellt worden [1]. Der oktaedrische Aufbau ist durch eine Röntgenstrukturanalyse gesichert [2]. Die an verschiedenen festen und gelösten Salzen registrierten IR- und Ramanspektren ließen sich entsprechend der $\mathrm{O}_{\mathrm{h}}$ Symmetrie zuordnen [3, 4]. Im Gegensatz zu den höheren Hydroboraten, von denen man sehr viele Substitutionsprodukte kennt, ist von $\mathrm{B}_{6} \mathrm{H}_{6}{ }^{2-}$ als einziges Derivat $\mathrm{B}_{6} \mathrm{Br}_{6}{ }^{2-}$ nur einmal erwähnt worden [5].

Unseres Wissens wird im folgenden erstmalig über die Darstellung der perhalogenierten closo-Boratanionen $\mathrm{B}_{6} \mathrm{X}_{6}{ }^{2-}, \mathrm{X}=\mathrm{Cl}, \mathrm{Br}, \mathrm{I}$, berichtet. Sie werden durch ihre ${ }^{11} \mathrm{~B}-\mathrm{NMR}$ - und Schwingungsspektren charakterisiert.

\section{Darstellung und Eigenschaften}

Bei der Umsetzung von $\mathrm{B}_{6} \mathrm{H}_{6}{ }^{2-}$ mit freien Halogenen in alkalischer Lösung erhält man über eine Folge von Substitutionsschritten Gemische von Halogenohydroboraten:

$$
\begin{aligned}
& \mathrm{B}_{6} \mathrm{H}_{6}{ }^{2-}+n \mathrm{X}_{2} \rightarrow \mathrm{X}_{n} \mathrm{~B}_{6} \mathrm{H}_{6-n}{ }^{2-}+n \mathrm{HX} \\
& \mathrm{X}=\mathrm{Cl}, \mathrm{Br}, \mathrm{I} ; n=1-6
\end{aligned}
$$

Für die Chlorierung bzw. Bromierung werden die Halogene in stark basischer, wäßriger Lösung ver-

\footnotetext{
* Sonderdruckanforderungen an Prof. Dr. Wilhelm Preetz.

$0340-5087 / 84 / 1100-1472 / \$ 01.00 / 0$
}

wendet, weil die entstehende Halogenwasserstoffsäure die Borate niedrigen Halogenierungsgrades und $\mathrm{B}_{6} \mathrm{H}_{6}{ }^{2-}$ sofort zersetzen würde. Zur Iodierung wird eine $\mathrm{I}_{2} / \mathrm{KI}$-Lösung mit einer basischen Lösung von $\mathrm{B}_{6} \mathrm{H}_{6}{ }^{2-}$ umgesetzt.

Mit großen Überschüssen der Halogene entstehen ausschließlich die Hexahalogenoborate. Sie ergeben mit Tetrabutylammonium(TBA)- oder Tetramethylammonium(TMA)-Ionen farblose Fällungen, aus denen durch Ionenaustausch die Alkalisalze erhalten werden. Die (TBA)-Salze lösen sich sehr gut in Dichlormethan, Acetonitril und Aceton, aber nicht in Wasser. Die Cs-Salze sind in Wasser schlecht, die Na-Salze dagegen gut löslich. Alle perhalogenierten Derivate von $\mathrm{B}_{6} \mathrm{H}_{6}{ }^{2-}$ sind in verdünnten Mineralsäuren längere Zeit stabil.

\section{${ }^{11} B$-NMR-Spektren}

$\mathrm{Da}$ in den oktaedrischen $\mathrm{B}_{6} \mathrm{X}_{6}{ }^{2-}$-Ionen alle Boratome symmetrieäquivalent sind, beobachtet man in den ${ }^{11} \mathrm{~B}$-NMR-Spektren (im Falle von $\mathrm{B}_{6} \mathrm{H}_{6}{ }^{2-}$ bei ${ }^{1} \mathrm{H}$ Entkopplung) jeweils nur ein scharfes Singulett. Die für $\mathrm{B}_{6} \mathrm{H}_{6}{ }^{2-}$ gemessene chemische Verschiebung $\delta=$ $-13,5 \mathrm{ppm}$ und die Kopplungskonstante $J\left({ }^{11} \mathrm{~B}-{ }^{1} \mathrm{H}\right)$ $=145 \mathrm{~Hz}$ stimmen annähernd mit den Literaturangaben überein [1]. Im Vergleich zu $\mathrm{B}_{12} \mathrm{H}_{12}{ }^{2-}$ erscheint das Signal von $\mathrm{B}_{6} \mathrm{H}_{6}{ }^{2-}$ um 1,4 ppm tieffeldverschoben.

Für die Abstufung $\mathrm{H}(\delta=-13,5 \mathrm{ppm})<\mathrm{Cl}(\delta=$ $-17,4 \mathrm{ppm})<\operatorname{Br}(\delta=-18,5 \mathrm{ppm})<\mathrm{I}(\delta=$ $-27.4 \mathrm{ppm}$ ) dürfte der in der gleichen Reihe zunehmende Anisotropieeffekt verantwortlich sein [6]. 


\section{Schwingungsspektren}

In Abb. 1 ist neben den IR- und Ramanspektren des festen $\mathrm{Cs}_{2} \mathrm{~B}_{6} \mathrm{H}_{6}$ zum Vergleich das Ramanspektrum der wäßrigen Lösung von $\mathrm{Na}_{2} \mathrm{~B}_{6} \mathrm{H}_{6}$, und zwar für parallele (II) bzw. senkrechte $(\perp)$ Stellung des Polarisationsfilters wiedergegeben. Abb. 2 zeigt die Schwingungsspektren der perhalogenierten Borate. Die genauen Frequenzen und die Zuordnungen der Schwingungen für verschiedene feste und in Wasser gelöste Salze sind in Tab. I zusammengestellt.

Für die Ionen $\mathrm{B}_{6} \mathrm{X}_{6}{ }^{2-}$ werden bei Annahme von $\mathrm{O}_{\mathrm{h}}$-Symmetrie die folgenden Grundschwingungen erwartet:

$$
2 \mathrm{~A}_{1 \mathrm{~g}}+2 \mathrm{E}_{\mathrm{g}}+\mathrm{F}_{1 \mathrm{~g}}+2 \mathrm{~F}_{2 \mathrm{~g}}+3 \mathrm{~F}_{1 \mathrm{u}}+2 \mathrm{~F}_{2 \mathrm{u}}
$$

Von diesen sind nach den Auswahlregeln die Schwingungen der Rassen $F_{1 g}$ und $F_{2 u}$ inaktiv, so daß in den Ramanspektren jeweils 6 Schwingungen gerader, in den IR-Spektren 3 ungerader Parität erwartet werden.

In Übereinstimmung mit der Analyse der Schwingungsspektren der beiden closo-Hydroborate
$\mathrm{B}_{10} \mathrm{H}_{10}{ }^{2-}$ und $\mathrm{B}_{12} \mathrm{H}_{12}{ }^{2-}$ sowie deren teilweise oder vollständig halogenierten Derivaten zeigt auch der Vergleich der IR- und Ramanspektren der Abb. 1 und 2 charakteristische Gruppenfrequenzen [7-11]. Das läßt auf eine nur geringe Kopplung der internen Schwingungen des $\mathrm{B}_{6}$-Käfigs mit solchen der Substituentenschale schließen. Tatsächlich findet man in allen Fällen im Bereich von $750-1250 \mathrm{~cm}^{-1}$ nahezu lagekonstante Banden, die eindeutig von dem $\mathrm{B}_{6}$-Gerüst herrühren. In den Ramanspektren der festen Cs-Salze weisen sie eine Strukturierung auf, die schon früher für $\mathrm{B}_{6} \mathrm{H}_{6}{ }^{2-}$ und für einige einfachere Carborane als Aufspaltung durch die Isotope ${ }^{10} \mathrm{~B}$ und ${ }^{11} \mathrm{~B}$ erklärt worden ist $[4,12]$. Damit erscheint es gerechtfertigt, eine Separierung der 9 Grundschwingungen vorzunehmen. Auf den $\mathrm{B}_{6}$-Käfig entfallen 4 interne Schwingungen der Rassen: $A_{1 g}+E_{g}+F_{1 u}+F_{2 g}$. Die verbleibenden 5 Schwingungen der Rassen: $\mathrm{A}_{1 \mathrm{~g}}+$ $\mathrm{E}_{\mathrm{g}}+2 \mathrm{~F}_{1 \mathrm{u}}+\mathrm{F}_{2 \mathrm{~g}}$ sind die eines regulären Oktaeders, in dem das Zentralion durch den $\left[\mathrm{B}_{6}\right]$-Cluster repräsentiert ist, der sich insbesondere gegenüber den schwereren Substituenten wie ein einziges Teilchen
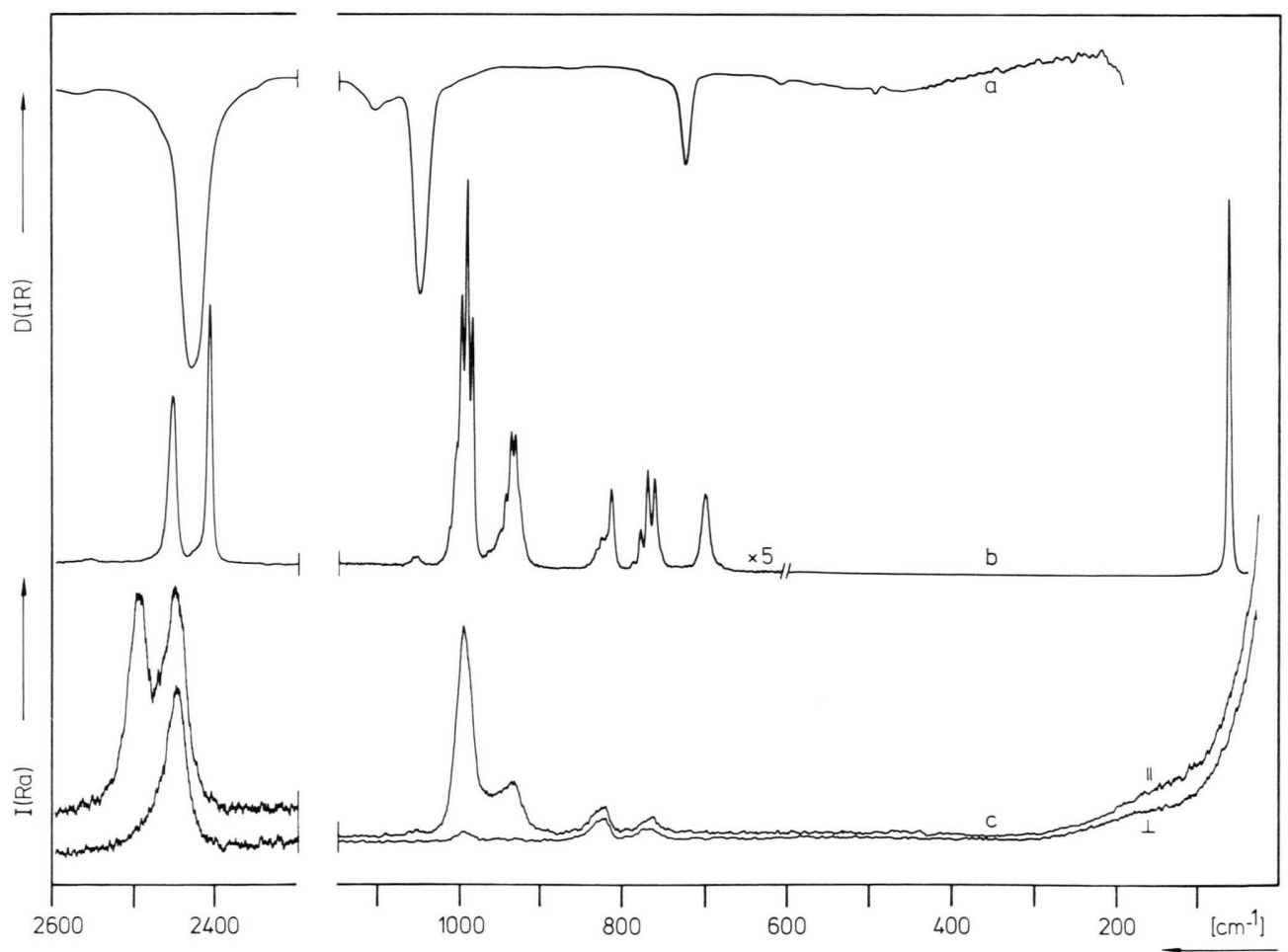

Abb. 1. IR-(a) und Ramanspektrum(b) des festen $\mathrm{Cs}_{2} \mathrm{~B}_{6} \mathrm{H}_{6}$; Ramanspektrum des in $\mathrm{H}_{2} \mathrm{O}$ gelösten $\mathrm{Na}_{2} \mathrm{~B}_{6} \mathrm{H}_{6}(\mathrm{c})$ bei paralleler (II) bzw. senkrechter $(\perp)$ Stellung des Polarisationsfilters. 
mittlerer Masse verhält. Bei der systematischen Numerierung werden für jede Punktgruppe die Gerüstschwingungen zuerst genannt, Tab. I.

Die Polarisationsmessungen der Ramanbanden von $\mathrm{B}_{6} \mathrm{H}_{6}{ }^{2-}$ machen einige Korrekturen der von Bragin et al. $[3,4]$ gegebenen Zuordnungen notwendig.
Von der Doppelbande im BH-Valenzschwingungsbereich ist die höherfrequente totalpolarisiert, die längerwelligere depolarisiert, Abb. 1. Die Zuordnung der ersteren als Atmungsschwingung der Substituentenschale $v_{2}$ und der zweiten zur zweifach entarteten symmetrischen $\mathrm{BH}-$ Valenzschwingung $v_{4}$ ist

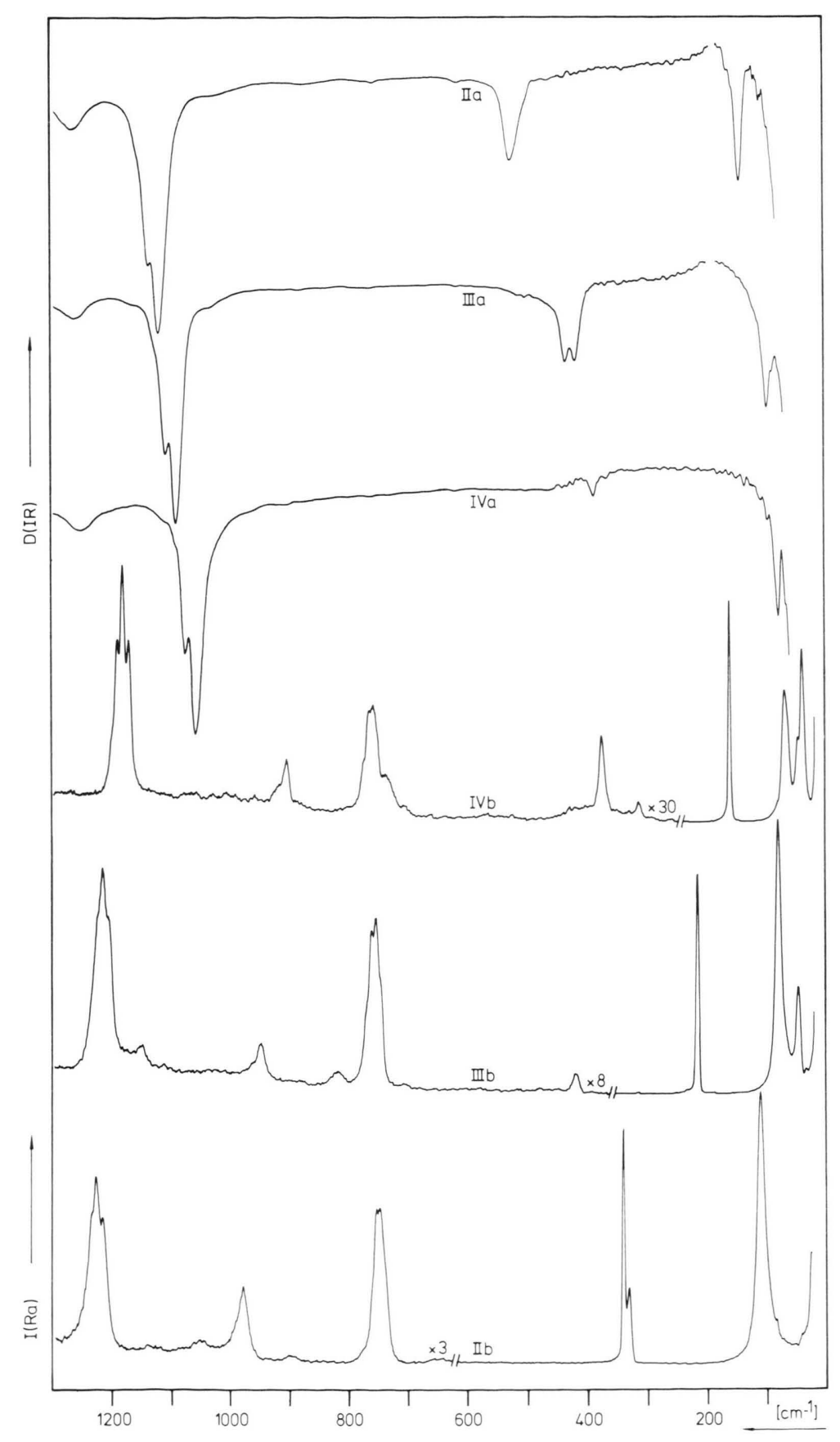

Abb. 2. IR-(a) und Ramanspektren(b) von festem $\mathrm{Cs}_{2} \mathrm{~B}_{6} \mathrm{Cl}_{6}$ (II), $\mathrm{Cs}_{2} \mathrm{~B}_{6} \mathrm{Br}_{6}$ (III) und $\mathrm{Cs}_{2} \mathrm{~B}_{6} \mathrm{I}_{6}(\mathrm{IV})$. 
Tab. I. Zuordnung und Frequenzen der IR- bzw. Ramanschwingungen $\left[\mathrm{cm}^{-1}\right]$ der festen Cs- bzw. in Wasser gelösten Naoder (TMA)-Salze von $\mathrm{B}_{6} \mathrm{X}_{6}{ }^{2-} ; \mathrm{X}=\mathrm{H}, \mathrm{Cl}, \mathrm{Br}, \mathrm{I}$.

\begin{tabular}{|c|c|c|c|c|c|c|c|c|c|c|c|c|c|}
\hline & $\begin{array}{l}\mathrm{Cs}_{2} \mathrm{~B}_{6} \mathrm{H} \\
\text { IR } \\
\text { (fest) }\end{array}$ & $\begin{array}{l}\mathrm{H}_{6} \\
\mathrm{Ra} \\
\text { (fest) }\end{array}$ & $\begin{array}{l}\mathrm{Na}_{2} \mathrm{~B}_{6} \mathrm{H}_{6} \\
\mathrm{Ra} \\
\left(\text { in } \mathrm{H}_{2} \mathrm{O}\right)\end{array}$ & $\begin{array}{l}(\mathrm{TMA})_{2} \mathrm{~B}_{6} \mathrm{H}_{6} \\
\mathrm{Ra} \\
\left(\text { in } \mathrm{H}_{2} \mathrm{O}\right)\end{array}$ & $\begin{array}{l}\mathrm{Cs}_{2} \mathrm{~B}_{6} \mathrm{C} \\
\mathrm{IR} \\
\text { (fest) }\end{array}$ & $\begin{array}{l}\mathrm{l}_{6} \\
\mathrm{Ra} \\
\text { (fest) }\end{array}$ & $\begin{array}{l}\mathrm{Na}_{2} \mathrm{~B}_{6} \mathrm{Cl}_{6} \\
\mathrm{Ra} \\
\left(\text { in } \mathrm{H}_{2} \mathrm{O}\right)\end{array}$ & $\begin{array}{l}\mathrm{Cs}_{2} \mathrm{~B}_{6} \\
\mathrm{IR} \\
\text { (fest) }\end{array}$ & $\begin{array}{l}3 \mathrm{r}_{6} \\
\mathrm{Ra} \\
\text { (fest) }\end{array}$ & $\begin{array}{l}\mathrm{Na}_{2} \mathrm{~B}_{6} \mathrm{Br}_{6} \\
\mathrm{Ra} \\
\left(\text { in } \mathrm{H}_{2} \mathrm{O}\right)\end{array}$ & $\begin{array}{l}\mathrm{Cs}_{2} \mathrm{~B}_{6} \mathrm{I}_{6} \\
\text { IR } \\
\text { (fest) }\end{array}$ & $\begin{array}{l}{ }^{6} \\
\mathrm{Ra} \\
\text { (fest) }\end{array}$ & $\begin{array}{l}\mathrm{Na}_{2} \mathrm{~B}_{6} \mathrm{I}_{6} \\
\mathrm{Ra} \\
\left(\text { in } \mathrm{H}_{2} \mathrm{O}\right)\end{array}$ \\
\hline$v_{3}: v(\mathrm{BB}), \mathrm{E}_{\mathrm{g}}$ & & $\begin{array}{l}932 \\
938 \\
944 \\
950\end{array}$ & 938 & $\begin{array}{l}939 \\
954^{\mathrm{a}}\end{array}$ & & 981 & 991 & & 947 & n. b. & & 902 & n. b. \\
\hline$v_{4}: v(\mathrm{BX}), \mathrm{Eg}$ & & 2412 & 2451 & 2452 & & n. b. & 328 & & n. b. & 208 & & n. b. & 155 \\
\hline$v_{6}: \delta\left(\left[\mathrm{B}_{6}\right] \mathrm{X}_{4}\right), \mathrm{F}_{2 \mathrm{~g}}$ & & $\begin{array}{l}\frac{814}{826} \\
833\end{array}$ & 824 & 820 & & n.b. & n. b. & & 38 & 38 & & 32 & 32 \\
\hline$v_{7}: v_{\mathrm{as}}(\mathrm{BB}), \mathrm{F}_{1 \mathrm{u}}$ & 2432 & & & & $\frac{1122}{1140}$ & & & $\frac{1091}{1108}$ & & & $\frac{1057}{1075}$ & & \\
\hline$v_{8}: v_{\text {as }}\left(\mathrm{X}\left[\mathrm{B}_{6}\right] \mathrm{X}\right), \mathrm{F}_{1 \mathrm{u}}$ & 1051 & & & & 527 & & & $\begin{array}{l}418 \\
438\end{array}$ & & & $\frac{380}{359}$ & & \\
\hline$v_{9}: \pi\left(\left[\mathrm{B}_{6}\right] \mathrm{X}_{4}\right), \mathrm{F}_{1 \mathrm{u}}$ & 731 & & & & 143 & & & 95 & & & 70 & & \\
\hline$v_{\mathrm{L}}$ & & 61 & & & & 108 & - & & 72 & - & & 62 & - \\
\hline
\end{tabular}

Hauptmaxima sind unterstrichen; n. b. = nicht beobachtet; ${ }^{a}$ Kationenbande.

damit gesichert. Die vermutete Aufspaltung durch Fermiresonanz ist auszuschließen [4]. Statt einer weiteren totalpolarisierten Bande treten davon zwei im Gerüstschwingungsbereich bei 994 und $938 \mathrm{~cm}^{-1}$ auf. Aus unerklärlichen Gründen sind diese von Bragin als depolarisiert und statt dessen diejenige bei $824 \mathrm{~cm}^{-1}$ als totalpolarisiert beschrieben worden, was dem vorgelegten experimentellen Befund widerspricht, Abb. 1. Eine überzeugende Erklärung für die vollständige Polarisation der beiden Banden 994/938 $\mathrm{cm}^{-1}$ kann derzeit nicht gegeben werden. Nach Intensität, Lage, dem charakteristischen Isotopenmuster und in Relation zu den entsprechenden Schwingungen in den Ramanspektren der perhalogenierten Verbindungen, Abb. 2, muß die Bande bei $994 \mathrm{~cm}^{-1}$ als $v_{1}$ angesehen werden. Gegen die Zuordnung der Schwingung bei $938 \mathrm{~cm}^{-1}$ als $v_{3}$ spricht der beobachtete Polarisationsgrad. Möglicherweise ist die Atmungsschwingung des Gerüstes aufgespalten und erscheint als Doppelbande. Die auch in den Hexahalogenoboraten relativ schwache Schwingung $v_{3}$ könnte mit dem starken Peak bei $994 \mathrm{~cm}^{-1}$ koinzidieren, unter dem bei senkrechter Stellung des Polarisationsfilters noch ein schwaches Signal erkennbar ist, Abb. 1.

Schwierig ist auch die Zuordnung der nahe beieinander liegenden depolarisierten Banden bei 824 und $767 \mathrm{~cm}^{-1}$. Da die geringste Kopplung mit Schwingungen der Substituenten für die Käfigdeformationsbewegungen $\mathrm{zu}$ erwarten ist, wird der Peak bei $767 \mathrm{~cm}^{-1}$, der im gleichen Bereich wie bei den perhalogenierten Verbindungen liegt, $v_{5}$ zugeordnet. Dafür spricht auch die ausgeprägte Isotopenstruktur. Die Deformationsschwingung $v_{6}$ bei $824 \mathrm{~cm}^{-1}$ sollte durch Deuterierung von $\mathrm{B}_{6} \mathrm{H}_{6}{ }^{2-}$ signifikant längerwellig verschoben werden, während $v_{5}$ davon kaum 
beeinflußt werden dürfte. Die im Ramanspektrum des festen $\mathrm{Cs}_{2} \mathrm{~B}_{6} \mathrm{H}_{6}$ bei $699 \mathrm{~cm}^{-1}$ auftretende Bande fehlt bei dem festen (TMA)- und bei allen in Wasser gelösten Salzen. Sie kann z.Z. nicht geklärt werden. Bei dem intensiven Peak bei $62 \mathrm{~cm}^{-1}$ handelt es sich um eine Gitterschwingung, die natürlich in den Lösungsspektren nicht auftritt.

Die Schwingungsspektren von $\mathrm{B}_{6} \mathrm{X}_{6}{ }^{2-}$ sind sehr ähnlich und zeigen für $\mathrm{X}=\mathrm{Cl}, \mathrm{Br}, \mathrm{I}$, systematische Verschiebungen. Bei den Gerüstschwingungen findet man für $v_{1}$ und $v_{5}$ aber auch für die IR-aktive antisymmetrische Valenzschwingung $v_{7}$ stärkere Isotopenaufspaltung. In den Ramanspektren ist $v_{1}$ stets intensiver als $v_{3}$; beide verschieben sich, wie auch $v_{7}$, mit schwerer werdendem Substituenten langwellig. Demgegenüber steigt die Frequenz der reinen Gerüstdeformationsschwingung $v_{5}$ von der $\mathrm{Cl}$ über die $\mathrm{Br}$ - zur I-Verbindung geringfügig an.

Im Vergleich zum steifen $\left[\mathrm{B}_{6}\right]$-Cluster wird die Substituentenschale in der Reihenfolge $\mathrm{Cl}, \mathrm{Br}$, I zunehmend deformierbarer, was auch in den hohen Ramanintensitäten zum Ausdruck kommt. Bei den festen Cs-Salzen von $\mathrm{B}_{6} \mathrm{X}_{6}{ }^{2-}$ fallen $v_{2}$ und $v_{4}$ praktisch zusammen. Die Aufspaltung bei $\mathrm{Cs}_{2} \mathrm{~B}_{6} \mathrm{Cl}_{6}$ ist vermutlich durch die Isotopen ${ }^{35} \mathrm{Cl}$ und ${ }^{37} \mathrm{Cl}$ bedingt. Die Polarisationsmessungen an Lösungen lassen erkennen, daß $v_{4}$ unter der totalpolarisierten $v_{2}-$ Schwingung verborgen ist, im Falle der $\mathrm{Br}$ - und IVerbindung aber um einige $\mathrm{cm}^{-1}$ von dieser abweicht.

Während der $\left[\mathrm{B}_{6}\right]$-Cluster an der Atmungsschwingung der Substituentenschale $v_{2}$ nicht teilnimmt, führt er bei der IR-aktiven Valenzschwingung $v_{8}$ als Ganzes antisymmetrische Bewegungen in der $\mathrm{X}_{6}$ Hülle aus. Für alle drei Hexahalogenoborate gilt $v_{8}>>v_{2}$. Da im Vergleich zu klassischen Oktaederkomplexen des Typs [ $\mathrm{MX}_{6}$ ] [13] die $\mathrm{X}_{6}$-Hülle um den $\left[\mathrm{B}_{6}\right]$-Cluster deutlich aufgeweitet ist, werden für die Deformationsschwingungen niedrige Frequenzen erwartet. Die IR-aktive out of plane-Schwingung $v_{9}$ wird an der Grenze des zugänglichen Meßbereichs gerade noch erfaßt. $v_{6}$ tritt dagegen nur in den Ramanspektren der I- bzw. Br-Verbindung als starke Bande zwischen 30 und $40 \mathrm{~cm}^{-1}$ auf. Im Gegensatz zu den ebenfalls sehr intensiven Gitterschwingungen findet man $v_{6}$ auch in den Lösungsspektren. Bei den im Ramanspektrum von $\mathrm{Cs}_{2} \mathrm{~B}_{6} \mathrm{I}_{6}$ bei 305 bzw. $370 \mathrm{~cm}^{-1}$ auftretenden Banden könnte es sich um den Oberton $2 v_{2}$ bzw. den Kombinationston $2 v_{2}+v_{\mathrm{L}}$ handeln. Entsprechend ist der Peak bei $415 \mathrm{~cm}^{-1}$ im
Ramanspektrum von $\mathrm{Cs}_{2} \mathrm{~B}_{6} \mathrm{Br}_{6}$ dem Oberton $2 v_{2}$ zuzuordnen.

\section{Experimentelles}

Die Darstellung von $\mathrm{Cs}_{2} \mathrm{~B}_{6} \mathrm{H}_{6}$ erfolgt nach [1]. Durch Behandeln mit einem entsprechend beladenen Kationenaustauscher werden daraus das $\mathrm{Na}$ bzw. (TMA)-Salz hergestellt.

\section{Darstellung der Hexahalogenoborate}

Chlorierung: $\mathrm{Zu}$ einer alkalischen Lösung von $200 \mathrm{mg} \mathrm{Na} \mathrm{B}_{6} \mathrm{H}_{6}$ in $100 \mathrm{ml}$ Wasser tropft man unter Rühren langsam eisgekühlte, mit $\mathrm{Cl}_{2}$ gesättigte $5 \mathrm{~N} \mathrm{NaOH}$. Die Reaktion ist beendet, wenn im BHValenzschwingungsbereich des IR-Spektrums einer kleinen Probefällung des (TMA)-Salzes keine Absorptionen mehr feststellbar sind. Bei Zugabe wäßriger $1 \mathrm{M}$ (TBA)Br-Lösung entsteht ein farbloser, flockiger Niederschlag. Durch Umkristallisieren aus Dichlormethan/Ether erhält man $(\mathrm{TBA})_{2} \mathrm{~B}_{6} \mathrm{Cl}_{6}$ in Form feinkristalliner Schuppen.

Bromierung: $\mathrm{Zu}$ der alkalischen Lösung von $200 \mathrm{mg} \mathrm{Na} \mathrm{B}_{6} \mathrm{H}_{6}$ in $100 \mathrm{ml}$ Wasser gibt man bei Raumtemperatur tropfenweise die orangegelbe Lösung von $\mathrm{Br}_{2}$ in $5 \mathrm{~N} \mathrm{NaOH}$, bis das Reaktionsgemisch eine schwache Gelbfärbung zeigt. Bei Zugabe von wäßriger $1 \mathrm{M}$ (TBA)Br-Lösung fällt (TBA) ${ }_{2} \mathrm{~B}_{6} \mathrm{Br}_{6}$ aus, das aus Dichlormethan/Ether umkristallisiert wird.

Iodierung: $\mathrm{Zu}$ einer schwach alkalischen Lösung von $200 \mathrm{mg} \mathrm{Na}{ }_{2} \mathrm{~B}_{6} \mathrm{H}_{6}$ in $100 \mathrm{ml}$ Wasser tropft man bei Raumtemperatur langsam eine wäßrige $c a$. 5proz. $\mathrm{I}_{2} / \mathrm{NaI}$-Lösung. Es ist darauf zu achten, daß das Gemisch durch gleichzeitige Zugabe von verd. $\mathrm{NaOH}$ stets schwach alkalisch bleibt. Die bleibende Gelbfärbung zeigt das Reaktionsende an. Das ausgefällte (TBA) ${ }_{2} \mathrm{~B}_{6} \mathrm{I}_{6}$ wird aus Dichlormethan/Ether umkristallisiert.

Cs- und Na-Salze: Aus den Lösungen von (TBA) ${ }_{2} \mathrm{~B}_{6} \mathrm{X}_{6} ; \mathrm{X}=\mathrm{Cl}, \mathrm{Br}, \mathrm{I}$, in Dichlormethan fallen bei Zugabe von $\mathrm{CH}_{3} \mathrm{COOCs} /$ Eisessig die in Wasser schwerlöslichen farblosen Salze $\mathrm{Cs}_{2} \mathrm{~B}_{6} \mathrm{X}_{6}$ mikrokristallin aus. Durch längeres Rühren in Gegenwart eines Kationenaustauschers in der $\mathrm{Na}^{+}$-Form lösen sich die Suspensionen der Cs-Salze in Wasser auf. Beim Eindampfen der Lösungen kristallisieren die in Wasser gut löslichen Salze $\mathrm{Na}_{2} \mathrm{~B}_{6} \mathrm{X}_{6}$ aus. Die Ausbeuten liegen etwa bei $75 \%$. Die Halogenidbestimmungen ergeben für

$$
\begin{array}{lll}
\mathrm{Cs}_{2} \mathrm{~B}_{6} \mathrm{Cl}_{6}: & \text { Ber. 39,14, } & \text { Gef. 38,3; } \\
\mathrm{Cs}_{2} \mathrm{~B}_{6} \mathrm{Br}_{6}: & \text { Ber. 59,18, } & \text { Gef. 58,5; } \\
\text { (TMA) }{ }_{2} \mathrm{~B}_{6} \mathrm{I}_{6}: & \text { Ber. 78,13, } & \text { Gef. 77,6\%. }
\end{array}
$$




\section{Spektren}

Die Messung der NMR-Spektren erfolgte bei $80,2 \mathrm{MHz}$ mit einem PFT-Spektrometer WM 250 der Fa. Bruker, Karlsruhe, welches mit einem Multikernprobenkopf und einer ${ }^{1} \mathrm{H}$-Breitbandentkopplungseinheit ausgestattet ist. Als Lösungsmittel und internes Locksignal wurde $\mathrm{CD}_{3} \mathrm{CN}$ verwendet. $\mathrm{BF}_{3} \cdot \mathrm{OEt}_{2}$ diente als externer Standard.

Die IR-Spektren wurden mit einem FT-IR-Spektrometer NIC 7199 der Fa. Nicolet, Offenbach/M., registriert. Dazu wurden für die Bereiche $200-600 \mathrm{~cm}^{-1}$ jeweils $10-40 \mathrm{mg}, 400-1350 \mathrm{~cm}^{-1}$ ca. $1 \mathrm{mg}$ und $2350-2650 \mathrm{~cm}^{-1} \mathrm{ca}$. $0,5 \mathrm{mg}$ der CsSalze mit 200 mg RbI verpreßt. Für den Bereich unterhalb $200 \mathrm{~cm}^{-1}$ eignen sich Preßlinge aus jeweils $200 \mathrm{mg}$ Polyethylen und ca. 20 mg der Cs-Salze.
Zur Aufnahme der Ramanspektren diente ein Cary 82 der Fa. Varian, Darmstadt. Die Festkörpermessungen wurden an den reinen feinkristallinen CsSalzen mit der Linie 514,5 nm eines Argon-Lasers bei einer Leistung von $500 \mathrm{~mW}$ durchgeführt. Die Registrierung der Ramanspektren an den wäßrigen kaltgesättigten Lösungen der $\mathrm{Na}$-Salze erfolgte in einer zylindrischen Quarzküvette entlang deren Achse bei einer auf $250 \mathrm{~mW}$ verminderten Leistung und unter Verwendung von Polarisationsfiltern.

Herrn Prof. Dr. H. C. Marsmann, Universität Gesamthochschule Paderborn, danken wir für die Registrierung der ${ }^{11} \mathrm{~B}-\mathrm{NMR}-\mathrm{Spektren}$, dem Fonds der Chemie für die Unterstützung mit Sachmitteln.
[1] J. L. Boone, J. Am. Chem. Soc. 86, 5036 (1964).

[2] R. Schaeffer, Q. Johnson und G. S. Smith, Inorg. Chem. 4, 917 (1965).

[3] J. Bragin und M. Diem, Proc. 5th. Intern. Conf. Raman Spectrosc., Freiburg i. Br., 88 (1976).

[4] J. Bragin, D. S. Urevig und M. Diem, J. Raman Spectrosc. 12, 86 (1982).

[5] W. R. Hertler nach F. Klanberg und E. L. Muetterties, Inorg. Chem. 5, 1955, Fußnote 17 (1966).

[6] H.-G. Srebny, W. Preetz und H.C. Marsmann, J. Org. Magn. Reson., im Druck.

[7] E. L. Muetterties, R. E. Merrifield, H. C. Miller, W. H. Knoth, Jr. und J. R. Downing, J. Am. Chem. Soc. 84, 4427 (1962).
[8] L. A. Leites, S. S. Bukalov, A. P. Kurbakova, M. M. Kaganski, Y. L. Gaft, N. T. Kuznetsov und J. A. Zakharova, Spectrochim. Acta 38a, 1047 (1982).

[9] L. A. Leites, A. P. Kurbakova, M. M. Kaganskii, Y. L. Gaft, I. A. Zakharova und N. T. Kuznetsov, Izv. Akad. Nauk SSSR, Ser. Khim. 1983, 2284.

[10] W. Preetz, H.-G. Srebny und H. C. Marsmann, Z. Naturforsch. 39b, 6 (1984).

[11] H.-G. Srebny, W. Preetz und H. C. Marsmann, Z. Naturforsch. 39b, 189 (1984).

[12] R. W. Jotham und D. J. Reynolds, J. Chem. Soc. A 1971, 3181

[13] W. Preetz, D. Ruf und D. Tensfeldt, Z. Naturforsch. 39b, 1100 (1984) 